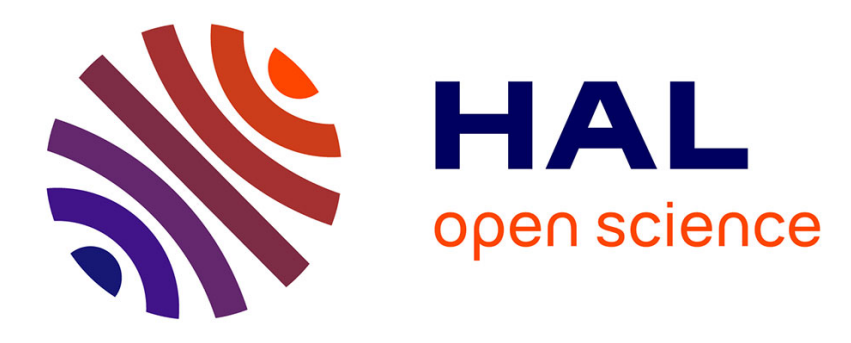

\title{
Bilan thermique des convertisseurs de l'énergie solaire: rôle de la sélectivité et de la concentration
}

\author{
A. Arconada, F. Papini
}

\section{To cite this version:}

A. Arconada, F. Papini. Bilan thermique des convertisseurs de l'énergie solaire: rôle de la sélectivité et de la concentration. Revue de Physique Appliquée, 1980, 15 (2), pp.163-167. 10.1051/rphysap:01980001502016300 . jpa-00244711

\section{HAL Id: jpa-00244711 https://hal.science/jpa-00244711}

Submitted on 1 Jan 1980

HAL is a multi-disciplinary open access archive for the deposit and dissemination of scientific research documents, whether they are published or not. The documents may come from teaching and research institutions in France or abroad, or from public or private research centers.
L'archive ouverte pluridisciplinaire HAL, est destinée au dépôt et à la diffusion de documents scientifiques de niveau recherche, publiés ou non, émanant des établissements d'enseignement et de recherche français ou étrangers, des laboratoires publics ou privés. 


\title{
Bilan thermique des convertisseurs de l'énergie solaire : rôle de la sélectivité et de la concentration
}

\author{
A. Arconada et F. Papini \\ Département d'Héliophysique, Université de Provence (*), \\ Centre de Saint-Jérôme, 13397 Marseille Cedex 4, France \\ (Reçu le 25 juin 1979, révisé le 29 octobre 1979, accepté le 31 octobre 1979)
}

\begin{abstract}
Résumé. - Le bilan thermique d'un convertisseur thermique de l'énergie solaire dépend de nombreux paramètres comme propriétés optiques de l'absorbeur, température de fonctionnement, concentration du rayonnement, coefficient de transfert convectif, etc...; l'influence de ces paramètres est difficile à estimer $a$ priori.

Dans cet exposé, on calcule les expressions analytiques du bilan, à partir d'une modélisation des propriétés optiques de la surface chaude. Par ailleurs, on expose une méthode permettant d'évaluer l'influence de chaque paramètre et utilisant la construction d'un diagramme particulier. On en traite un exemple d'application consistant en l'étude des variations de la sélectivité avec la température de fonctionnement de l'absorbeur solaire.
\end{abstract}

\begin{abstract}
The thermal balance of solar converters depends on numerous parameters, as : absorber optical properties, working temperature, radiation concentration, convection transfer coefficient, etc...

In this paper, the balance analytical expressions are calculated starting from modelizing hot surface optical properties. Elsewhere, a method is given that allows evaluation of each parameter influence, and which is based upon a specific chart. An example is presented that concerns the study of selectivity variation according to the solar absorber working temperature.
\end{abstract}

1. Introduction. - La conception d'un convertisseur thermique de l'énergie solaire nécessite une analyse $a$ priori de son bilan thermique compte tenu des conditions d'utilisation. Une telle analyse ne peut être effectuée qu'à partir d'un modèle permettant d'évaluer l'influence des divers paramètres caractéristiques du dispositif. C'est dans cet objectif que nous avons effectué une étude générale du bilan thermique d'un convertisseur faisant, en particulier, intervenir les propriétés de sélectivité de la surface absorbante et la concentration du rayonnement solaire. Nous décrivons ainsi la constitution d'abaques, conduisant à la détermination d'un des types de capteurs à utiliser, en vue de résoudre un problème de conversion donné.

2. Le bilan thermique. - Comme nous l'avons déjà exposé par ailleurs $[1,2]$, en toute généralité, les propriétés optiques de la surface absorbante peuvent être modélisées par le graphe de la figure 1, représentant la variation du facteur d'absorption ou d'émission monochromatique en fonction de la longueur d'onde.

(*) Equipe de Recherche associé au C.N.R.S. no 538.

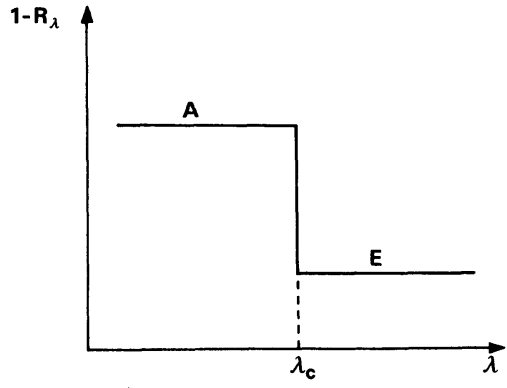

Fig. 1. - Modélisation des propriétés optiques de l'absorbeur : variation du facteur d'absorption monochromatique avec la longueur d'onde.

[Modelization of absorber optical properties : monochromatic absorptivity variation versus wavelength.]

Nous appellerons facteur de sélectivité le rapport $A / E=s$. Si $T$ est la température de surface de l'absorbeur et $T_{\text {en }}$ la température équivalente à son environnement, on peut écrire le bilan thermique :

$$
\varphi_{\mathrm{u}}+\varepsilon \sigma T^{4}+P_{\mathrm{c}}=\alpha C H+\alpha^{\prime} \sigma T_{\mathrm{en}}^{4}
$$

où

$P_{\mathrm{c}}$ représente les pertes par convection naturelle ou forcée et par conduction, 
$\varphi_{\mathrm{u}}$ la puissance thermique utilisable,

$\&$ le facteur d'émission total hémisphérique de l'absorbeur,

$\alpha$ son facteur d'absorption pour le spectre solaire,

$\alpha^{\prime}$ son facteur d'absorption pour les radiations émises par l'environnement,

$\mathrm{CH}$ le produit de la puissance incidente $H$ par le facteur de concentration géométrique $C$.

Le rendement instantané devient alors :

$$
\begin{array}{r}
\eta=\frac{\varphi_{u}}{C H}=\alpha\left[1-\frac{\varepsilon}{\alpha} \frac{\sigma\left(T^{4}-T_{\mathrm{en}}^{4}\right)}{C H}\right]+ \\
\quad+\frac{\left(\alpha^{\prime}-\varepsilon\right) \sigma T_{\mathrm{en}}^{4}}{C H}-\frac{P_{\mathrm{c}}}{C H} .
\end{array}
$$

Dans la plupart des cas, l'inégalité

$$
\left|\frac{\varepsilon}{\varepsilon-\alpha^{\prime}}\right| \gg\left|\frac{T_{\mathrm{en}}^{4}}{T^{4}-T_{\mathrm{en}}^{4}}\right|
$$

est vérifiée $\left({ }^{1}\right)$, et cette relation se simplifie, soit :

$$
\eta=\alpha\left[1-\frac{\varepsilon}{\alpha} \frac{\sigma\left(T^{4}-T_{\mathrm{en}}^{4}\right)}{C H}\right]-\frac{P_{\mathrm{c}}}{C H} .
$$

Si l'on ne tient pas compte des pertes par convection et conduction, on obtient le rendement radiatif instantané :

avec

$$
\eta_{\mathrm{R}}=\alpha\left[1-\frac{\varepsilon}{\alpha} \frac{\sigma\left(T^{4}-T_{\mathrm{en}}^{4}\right)}{C H}\right]
$$

$$
\eta=\eta_{\mathrm{R}}-\frac{P_{\mathrm{c}}}{C H}
$$

En assimilant le rayonnement solaire et celui de l'environnement à l'émission de corps noirs portés respectivement aux températures $T_{\mathrm{s}}$ et $T_{\mathrm{en}}$, on peut calculer, avec le schéma de la figure 1 :

où

$$
\left\{\begin{array}{l}
\alpha=(A-E) k_{\mathrm{s}}+E \\
\varepsilon=(A-E) k+E \\
\alpha^{\prime}=(A-E) k_{\mathrm{en}}+E
\end{array}\right.
$$

$$
\begin{aligned}
k_{\mathrm{s}} & =\frac{\int_{0}^{\lambda_{\mathrm{c}}} M\left(\lambda, T_{\mathrm{s}}\right) \mathrm{d} \lambda}{\sigma T_{\mathrm{s}}^{4}} \\
k_{\mathrm{en}} & =\frac{\int_{0}^{\lambda_{\mathrm{c}}} M\left(\lambda, T_{\mathrm{en}}\right) \mathrm{d} \lambda}{\sigma T_{\mathrm{en}}^{4}} \\
k & =\frac{\int_{0}^{\lambda_{\mathrm{c}}} M(\lambda, T) \mathrm{d} \lambda}{\sigma T^{4}}
\end{aligned}
$$

( $\left.{ }^{1}\right)$ On peut démontrer que l'erreur introduite par cette simplification ne dépasse pas $2 \%$ si $\lambda_{\mathrm{c}}<3 \mu \mathrm{m}$ (voir Fig. 1).
$M\left(\lambda, T_{\mathrm{s}}\right), M\left(\lambda, T_{\mathrm{en}}\right)$ et $M(\lambda, T)$ étant les émittances monochromatiques de corps noirs pour les températures $T_{\mathrm{s}}, T_{\text {en }}$ et $T$.

De façon générale, le facteur $k_{\mathrm{i}}$ est égal à la proportion de puissance émise par un corps noir à la température $T_{\mathrm{i}}$, dans la bande de longueur d'onde $0-\lambda_{\mathrm{c}}$. On déduit de ces expressions :

$$
\begin{aligned}
\eta_{\mathrm{R}}=A\left[\left(\left(1-\frac{1}{s}\right) k_{\mathrm{s}}+\frac{1}{s}\right)-\right. & \\
& \left.\left(\left(1-\frac{1}{s}\right) k+\frac{1}{s}\right) \frac{\sigma\left(T^{4}-T_{\mathrm{en}}^{4}\right)}{C H}\right] .
\end{aligned}
$$

Il faut noter que le facteur monochromatique $A$ intervient comme coefficient multiplicatif du rendement. On peut donc aussi poser :

$$
\eta_{\mathrm{R}} / A=K_{1}-\frac{K_{2}}{s}
$$

avec :

$$
\left\{\begin{array}{l}
K_{1}=k_{\mathrm{s}}-k \frac{\sigma\left(T^{4}-T_{\mathrm{en}}^{4}\right)}{C H} \\
K_{2}=(.1-k) \frac{\sigma\left(T^{4}-T_{\mathrm{en}}^{4}\right)}{C H}-\left(1-k_{\mathrm{s}}\right) .
\end{array}\right.
$$

Lorsque le facteur de sélectivité est infini, le rendement radiatif est maximum, soit :

$$
\left[\eta_{\mathrm{R}} / A\right]_{\max }=K_{1} \text {. }
$$

Dans le cas où la longueur d'onde de coupure $\lambda_{\mathrm{c}}$ est optimisée, c'est-à-dire lorsqu'elle est choisie pour que :

$$
\left(\frac{\partial \eta_{\mathrm{R}} / A}{\partial \lambda_{\mathrm{c}}}\right)_{\lambda_{\mathrm{co}}}=0
$$

on obtient une relation uniquement fonction de $\lambda_{\text {co }}$ (longueur d'onde de coupure optimisée), $\mathrm{CH}, \mathrm{T}$ et $T_{\text {en }}$ [2], donnée par :

$C H=\sigma\left(T^{4}-T_{\mathrm{en}}^{4}\right) \quad \frac{T_{\mathrm{s}}^{4}}{T^{4}} \frac{\exp \frac{C_{2}}{\lambda_{\mathrm{co}} T_{\mathrm{s}}}-1}{\exp \frac{C_{2}}{\lambda_{\mathrm{co}} T}-1}$

avec :

$$
\begin{aligned}
C_{2} & =14,39 \times 10^{-3} \mathrm{~m} \cdot \mathrm{K} \\
\sigma & =5,67 \times 10^{-8} \mathrm{~W} \cdot \mathrm{m}^{-2} \cdot \mathrm{K}^{-4}
\end{aligned}
$$

la valeur de $\lambda_{\text {co }}$ est donc indépendante du rapport $A / E$.

A partir des relations ainsi posées, il est possible de construire des abaques permettant de déterminer un système de conversion pour une utilisation donnée, c'est-à-dire pour une température de fonctionnement $T$.

3. Choix des paramètres caractéristiques d'un convertisseur. - Ce choix porte sur les paramètres suivants : $\lambda_{\mathrm{c}}, A / E$ et $C$. Comme on vient de le voir, la 
valeur de la longueur d'onde de coupure à prendre en considération est $\lambda_{\text {co }}$, c'est-à-dire celle qui induit le plus haut rendement. Dans ces jonditions, $\lambda_{\text {co }}$ est une fonction $g_{1}$ de $C H$ et de $T$, soit :

et donc :

$$
\lambda_{\mathrm{co}}=g_{1}(\mathrm{CH}, \mathrm{T})
$$

$$
\begin{aligned}
& \left(\eta_{\mathrm{R}} / A\right)_{\max }=g_{2}(C H, T) \\
& K_{2}=g_{3}(C H, T) .
\end{aligned}
$$

Par conséquent :

$$
\eta_{\mathrm{R}} / A=g_{4}(C H, T, s) .
$$

Pour une température $T$ donnée, le rendement effectif du convertisseur est en fait égal au rendement maximum $\left(\eta_{\mathrm{R}} / A\right)_{\max }$ multiplié par $\gamma$ coefficient de performance, nombre réel variant entre 0 et 1 (puisque s est un nombre fini). On aura donc :

$$
\left(\eta_{\mathrm{R}} / A\right)_{\max }=g_{2}(C H) \quad \text { et } \quad \eta_{\mathrm{R}} / A=\gamma g_{2}(C H) .
$$

Le rendement maximum qu'il est possible d'atteindre avec un convertisseur thermique est fonction principalement de la puissance incidente. Le choix de cette valeur étant effectué, on trouve :

$$
\gamma=g_{5}(s) \text {. }
$$

Autrement dit, l'utilisation de la sélectivité ne sert qu'à obtenir un rendement effectif plus ou moins proche du rendement maximum. Le facteur de sélectivité détermine la valeur de $\gamma$, alors que la concentration fixe celle du rendement maximum du dispositif.

On obtient donc l'expression :

$$
s(1-\gamma)=\frac{K_{2}}{K_{1}} .
$$

Soit, en remplaçant $K_{1}$ et $K_{2}$ par leur valeur :

$(1-\gamma) s\left(\eta_{\mathrm{R}} / A\right)_{\max }+1-\left(\eta_{\mathrm{R}} / A\right)_{\max }=\frac{\sigma\left(T^{4}-T_{\mathrm{en}}^{4}\right)}{C H}$

Dans le cas où l'on prend en compte les pertes par convection et conduction, on peut poser :

$$
P_{\mathrm{c}}=h\left(T-T_{\mathrm{en}}\right)
$$

en linéarisant ces pertes par rapport à la température $T_{\text {en }}$.

On a alors :

$$
\eta / A=K_{1}-\frac{K_{2}}{s}-\frac{h\left(T-T_{\mathrm{en}}\right)}{A C H}
$$

d'où :

$$
(\eta / A)_{\max }=K_{1}-\frac{h\left(T-T_{\mathrm{en}}\right)}{A C H}
$$

On peut en déduire la nouvelle relation :

$$
\begin{aligned}
(1-\gamma) s(\eta / A)_{\max }+1 & -(\eta / A)_{\max }= \\
& =\frac{\sigma\left(T^{4}-T_{\mathrm{en}}^{4}\right)}{C H}+\frac{h\left(T-T_{\mathrm{en}}\right)}{A C H} .
\end{aligned}
$$

Si l'on définit la grandeur

$$
y=(1-\gamma) s(\eta / A)_{\max }+1-(\eta / A)_{\max },
$$

on obtient la relation :

$$
\begin{array}{rl}
\log _{10} y=-\log _{10} & C H+\log _{10} \\
\times & \left(\sigma\left(T^{4}-T_{\mathrm{en}}^{4}\right)+\frac{h\left(T-T_{\mathrm{en}}\right)}{A}\right) .
\end{array}
$$

La variation de $\log _{10} y$ dépend linéairement $\mathrm{du}$ $\log _{10} C H$. Les courbes représentatives sont tracées sur les figures 2, 3, 4 et 5 ; elles sont obtenues pour des valeurs de $h / A$ égales à $0,1,5$ et $10 \mathrm{~W} \cdot \mathrm{m}^{-2} \cdot{ }^{\circ} \mathrm{C}^{-1}$. Par ailleurs, on a porté sur ces diagrammes les courbes $(\eta / A)_{\max }=$ Cte, calculées à partir de graphes du type de celui donné figure 6 . De tels abaques permettent de calculer les caractéristiques d'un convertisseur pour une température $T$ fixée. Ils contiennent en effet toutes les grandeurs nécessaires à cette détermination. Un exemple d'emploi de ce type d'abaque peut être donné; cherchons la valeur de $s$, nécessaire à une conversion thermique utilisant un miroir concentrateur cylindro-parabolique $(C=25)$, la température moyenne de surface de l'absorbeur étant $350^{\circ} \mathrm{C}$ et la puissance solaire instantanée $750 \mathrm{~W} . \mathrm{m}^{-2}$. On admettra $h / A=10 \mathrm{~W} \cdot \mathrm{m}^{-2} \cdot{ }^{\circ} \mathrm{C}^{-1}$, ce qui est un ordre de grandeur acceptable pour la convection naturelle autour du tube horizontal rectiligne, parcouru par un fluide caloporteur et placé au foyer du dispositif. La figure 5 permet de calculer, à partir de la courbe $(f)$, $\log _{10} y \simeq-0,2$ avec $(\eta / A)_{\max } \simeq 0,8$. L'expression de $y$ conduit à la relation $s(1-\gamma) \simeq 0,54$; si l'on désire que $\eta / A=0,7$ on en déduit $\gamma=0,875$ et $s \simeq 4,5 ; \operatorname{si} \eta / A=0,75$ on trouve $s \simeq 8,5$.

Pour une température $T$ donnée, on peut également calculer la valeur de $s$, en fonction de $\mathrm{CH}$, si l'on impose le rendement effectif. Sur la figure 7 sont représentées les variations du facteur de sélectivité pour $\eta / A=0,6, T$ étant égal à $150^{\circ} \mathrm{C}$ et $550^{\circ} \mathrm{C}$ :

- Pour un capteur plan (zone $\mathrm{Pl}$ ), on observe que la température de fonctionnement ne peut pas atteindre $150^{\circ} \mathrm{C}$ si les pertes par convection ne sont pas notablement réduites; même si les pertes $P_{\mathrm{c}}$ sont faibles, la valeur de $s$ doit être de l'ordre de 10 : c'est pratiquement la limite de la conversion thermique à l'aide de capteur plan sans concentration du rayonnement solaire.

- Pour $T=550^{\circ} \mathrm{C}$, les faibles concentrations comprises entre 1 et 25 (zone $\mathrm{CP}$, cas du cylindroparabolique) conduisent à l'utilisation d'un facteur de sélectivité qui peut être élevé, avec une influence importante de la convection forcée; l'élimination de tels inconvénients ne peut être réalisée qu'en se plaçant dans un domaine de puissance incidente correspondant à une concentration supérieure à 25 (zone $\mathrm{P}$, cas du miroir segmenté, approximation d'un paraboloïde de révolution, dont le facteur de concentration géométrique varie avec la taille relative des miroirs 


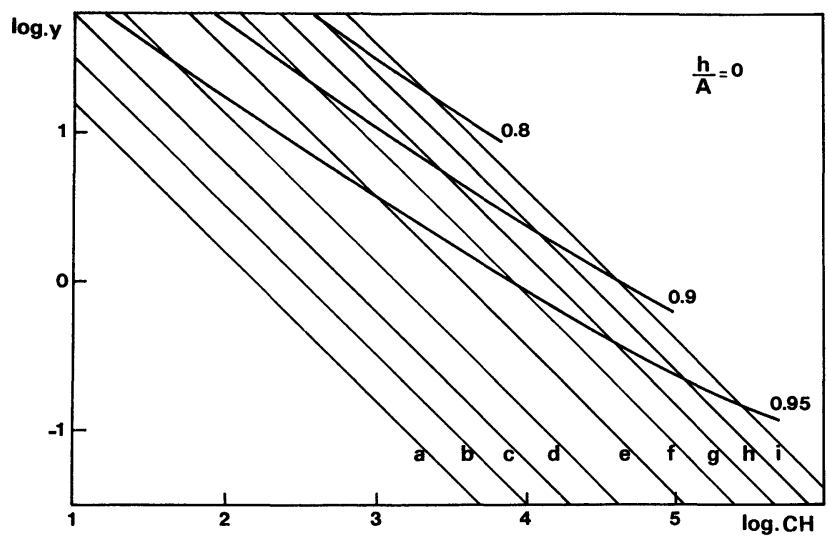

FIG. 2.

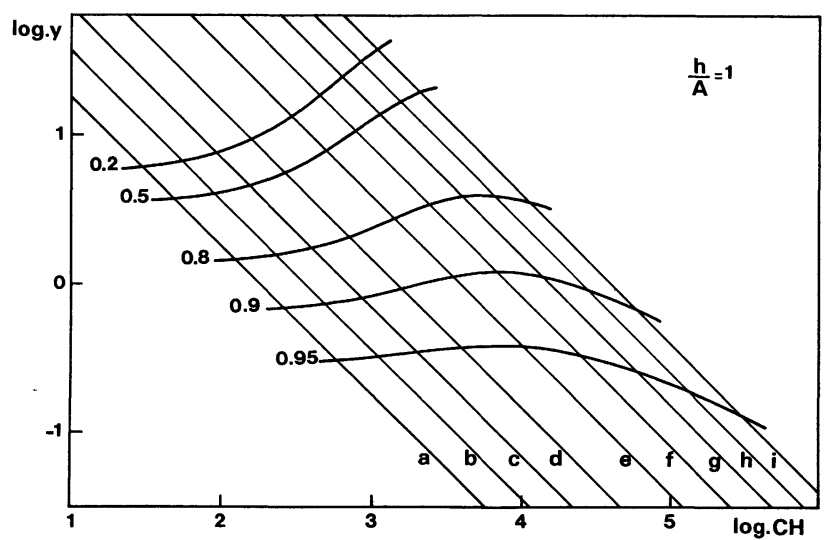

FIG. 3.
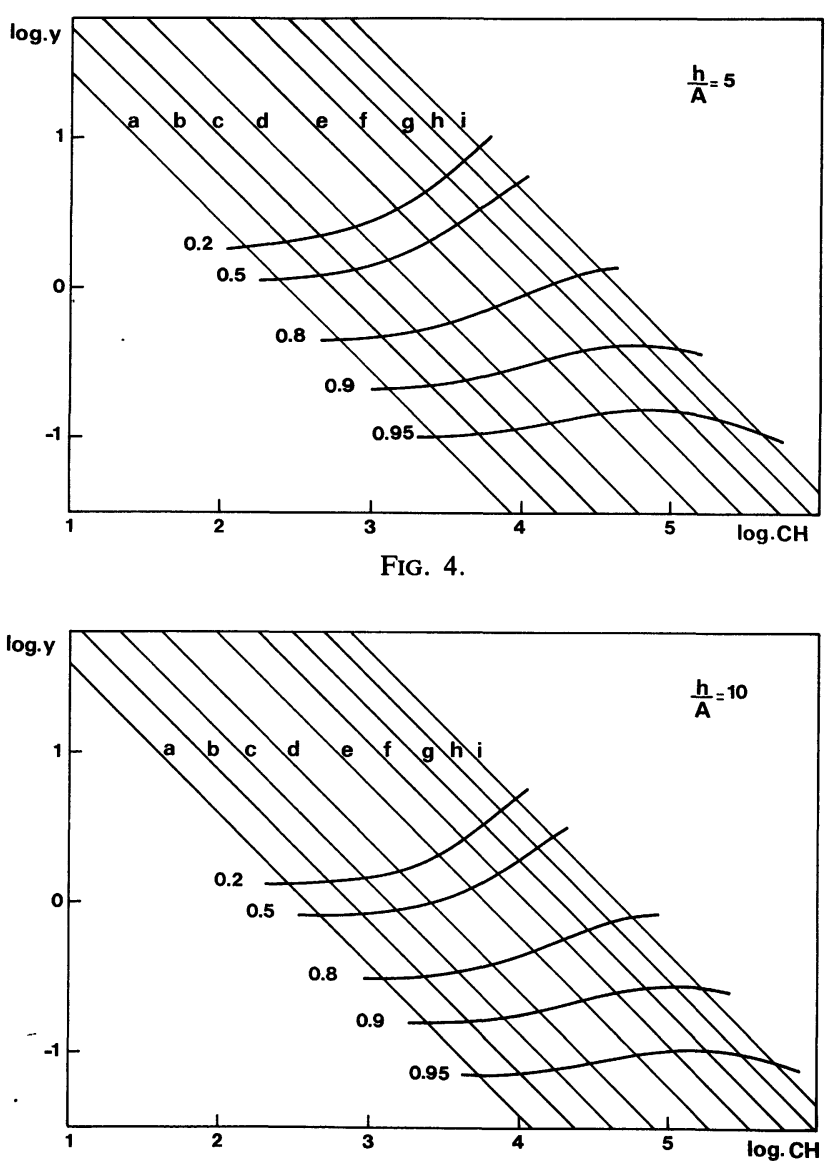

FIG. 5.

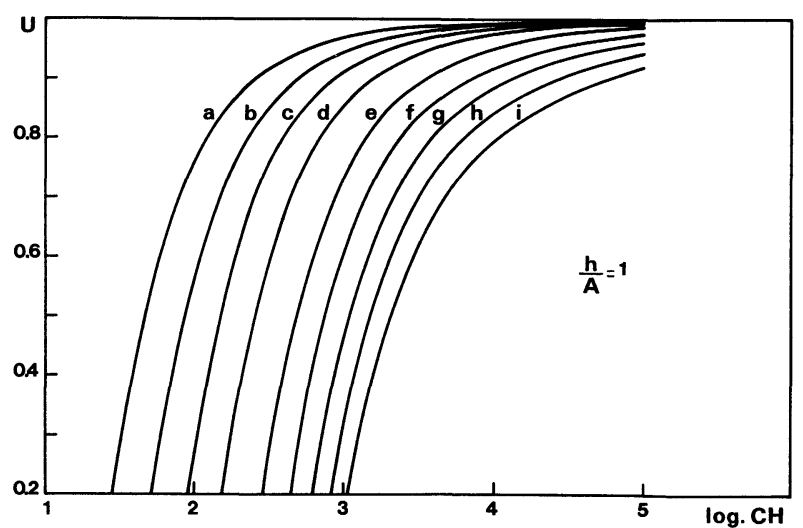

Fig. 6. - Courbes représentatives de la fonction

$U=(\eta / A)_{\max }=f\left(\log _{10} C H\right)$ pour $h / A=1 \mathrm{~W} \cdot \mathrm{m}^{-2} \cdot{ }^{\circ} \mathrm{C}^{-1}$.

[Curves representing function

$U=(\eta / A)_{\max }=f\left(\log _{10} C H\right)$ for $\left.h / A=1 \mathrm{~W} \cdot \mathrm{m}^{-2} \cdot{ }^{\circ} \mathrm{C}^{-1} \cdot\right]$

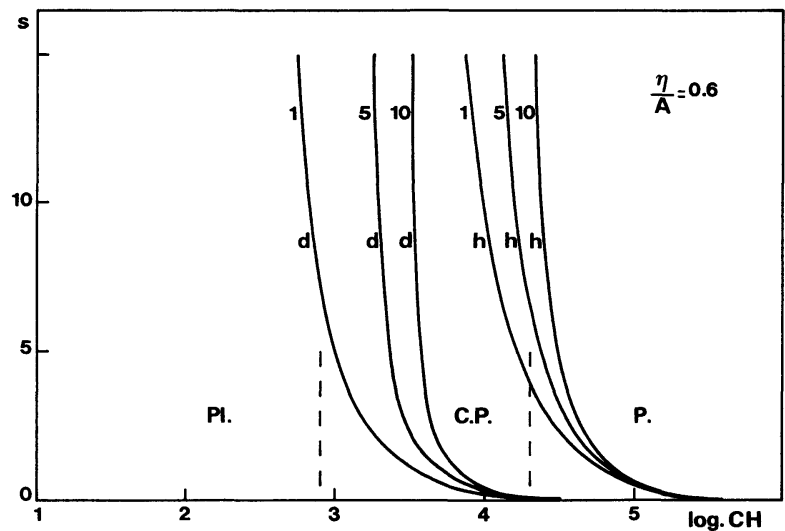

Fig. 7. - Variations du facteur de sélectivité $s$ en fonction de $\log _{10} C H$ pour deux températures $150^{\circ} \mathrm{C}$ et $550^{\circ} \mathrm{C}(d$ et $h), h / A$ étant égal à $1,5,10 \mathrm{~W} \cdot \mathrm{m}^{-2} \cdot{ }^{\circ} \mathrm{C}^{-1}$ et $\eta / A=0,6$.

[Selectivity $(s)$ variations, as a function of $\log _{10} \mathrm{CH}$ corresponding to two temperature levels $150^{\circ} \mathrm{C}$ and $550^{\circ} \mathrm{C}(d$ and $h), h / A$ being $1.5,10 \mathrm{~W} \cdot \mathrm{m}^{-2}$ and $\eta / A=0.6$.]

élémentaires [3]); le facteur de sélectivité diminue alors et devient même égal à 1 (cas de la surface grise ou noire). On note que lorsque la température $T$ augmente, il faut faire appel à des concentrateurs qui

Fig. 2, Fig. 3, Fig. 4, Fig. 5. - Droites représentatives de la fonction $\log _{10} y=f\left(\log _{10} C H\right)$, avec

$$
y=s(1-\gamma)(\eta / A)_{\max }+1-(\eta / A)_{\max } .
$$

$\mathrm{a}-50{ }^{\circ} \mathrm{C}, \mathrm{b}-70^{\circ} \mathrm{C}, \mathrm{c}-100^{\circ} \mathrm{C}, \mathrm{d}-150^{\circ} \mathrm{C}, \mathrm{e}-250^{\circ} \mathrm{C}, \mathrm{f}-350^{\circ} \mathrm{C}, \mathrm{g}-450^{\circ} \mathrm{C}$, $\left.\mathrm{h}-550^{\circ} \mathrm{C}, \mathrm{i}-650^{\circ} \mathrm{C}\right)$. On a porté également les courbes $(\eta / A)_{\max }=\mathrm{Cte}$ avec les valeurs correspondantes. On a pris $T_{\text {en }}=300 \mathrm{~K}$.

[Representation of the fonction $\log _{10} y=f\left(\log _{10} C H\right)$, with $y=s(1-\gamma)(\eta / A)_{\max }+1-(\eta / A)_{\max }\left(\mathrm{a}-50^{\circ} \mathrm{C}, \mathrm{b}-70^{\circ} \mathrm{C}, \mathrm{c}-100^{\circ} \mathrm{C}\right.$, $\left.\mathrm{d}-150^{\circ} \mathrm{C}, \mathrm{e}-250^{\circ} \mathrm{C}, \mathrm{f}-350^{\circ} \mathrm{C}, \mathrm{g}-450^{\circ} \mathrm{C}, \mathrm{h}-550^{\circ} \mathrm{C}, \mathrm{i}-650^{\circ} \mathrm{C}\right)$. Curves $(\eta / A)_{\max }=$ Constant are also given with corresponding values. $T_{\text {en }}=300 \mathrm{~K}$ has been taken.] 
ne peuvent être réalisés qu'à l'aide de systèmes à foyer quasi ponctuels; il est alors possible de diminuer la surface de la tache focale en augmentant la précision du dispositif, sans en changer le principe [3]. L'accroissement du facteur de concentration $C$ peut donc être préférable à l'emploi d'une surface sélective. A cette remarque d'ordre économique et technique, on peut adjoindre une justification purement physique. Pour cela, considérons une température suffisamment haute, $T=650^{\circ} \mathrm{C}$, et évaluons le facteur de sélectivité $s$; afin que l'absorption pour tout le spectre solaire soit correcte, il faut que $\lambda_{\text {co }} \simeq 2,5 \mu \mathrm{m}$, ce qui entraîne d'après la relation (2) $C H \simeq 2 \times 10^{5} \mathrm{~W} \cdot \mathrm{m}^{-2}$. Le rendement radiatif effectif étant pris tel que $\eta_{\mathrm{R}} / A=0,8$, on est conduit, avec l'expression (1), à $s \simeq 1$. Autrement dit, à très haute température, l'effort consenti pour avoir une longueur d'onde de coupure $\lambda_{\text {co }} \simeq 2,5 \mu \mathrm{m}$ induit une diminution de $s$ qui peut aller jusqu'à l'unité; l'égalité (2) permet de constater, si $\lambda_{\text {co }}$ est fixée à $2,5 \mu \mathrm{m}$, que le terme

$$
\sigma\left(T^{4}-T_{\mathrm{en}}^{4}\right) / C H
$$

décroît lorsque $T$ augmente. L'expression de $\eta / A$ peut alors s'écrire :

$$
\eta / A \simeq\left(1-\frac{1}{s}\right) k_{\mathrm{s}}+\frac{1}{s}, \text { avec } k_{\mathrm{s}} \simeq 1 .
$$

Le facteur de sélectivité n'intervenant plus, la solution la plus simple correspond à $s=1$. Evidemment, on peut toujours utiliser une surface sélective, de même qu'une concentration du rayonnement, quelle que soit la valeur de $T$, qui permettent aux faibles flux d'accroître le rendement de l'appareillage. Cependant, ce type d'utilisation n'a pas lieu d'être pris en considération au niveau d'un principe de conception; il représente plutôt un perfectionnement dont l'avantage devient faible si la valeur de $C$ est calculée en tenant compte des conditions moyennes d'ensoleillement sur le lieu d'implantation du convertisseur. La limite en température des domaines d'emploi de sélectivité et concentration et concentration est naturellement assez floue; on peut la situer aux environs de $500^{\circ} \mathrm{C}$, c'est-à-dire lorsque le spectre d'émission du corps chauffé est tel qu'une partie non négligeable de la puissance perdue (de l'ordre de $10 \%$ ) est comprise dans la bande spectrale définie par $\lambda<2,5 \mu \mathrm{m}$.

Cette analyse montre qu'il est possible de donner des limites aux conversions thermiques à moyenne et haute température, reposant sur des critères physiques. Ainsi, on peut dire que la moyenne température correspond aux dispositifs non équipés de systèmes de concentration du rayonnement, et pour lesquels on fait appel à des surfaces absorbantes sélectives $\left(70^{\circ} \mathrm{C}<T<150^{\circ} \mathrm{C}\right)$. Ensuite, on atteint la haute température dont la borne supérieure se situe aux environs de $500{ }^{\circ} \mathrm{C}$; pour cet autre domaine, on peut utiliser à la fois la concentration et la sélectivité. $\mathrm{Au}$-delà, pour la très haute température, la concentration devient le paramètre prépondérant.

4. Conclusions. - Nous avons donné au cours de cet exposé les relations générales permettant de calculer le rendement d'un convertisseur thermique de l'énergie solaire. Nous avons également donné le principe de construction d'un diagramme en vue d'examiner les influences de l'ensemble des paramètres caractéristiques du convertisseur. Ainsi, nous avons pu montrer comment intervient en particulier le facteur de sélectivité.

Il est également possible, avec ce type d'analyse, de choisir le point ou la courbe de fonctionnement de l'appareil, son utilisation imposant des conditions sur la température.

\section{Bibliographie}

[1] Pasquetti, R., Papini, F., Gallet, P., Revue Gén. Therm. XV, 169 (1976) 11-17.

[2] Pasquetti, R., Papini, F., Sol. Energy, vol. 21 (1978) 129.

[3] Pasquetti, R., Peri, G., Revue Phys. Appl. (à paraître). 